This is a post-peer-review, pre-copyedit version of an article published in the Journal of Autism and Developmental Disorders. The final authenticated version is available online at: https://doi.org/10.1007/s10803-020-04735-6

\author{
Brief Report: Mobile Technology to Support Parents in Reducing Stereotypy \\ Lydia Trudel $^{1}$, Marc J. Lanovaz ${ }^{1,2}$, and Isabelle Préfontaine ${ }^{1}$ \\ ${ }^{1}$ École de psychoéducation, Université de Montréal \\ ${ }^{2}$ Centre de recherche de l'Institut universitaire en santé mentale de Montréal
}

Conflict of interest: Lydia Trudel and Isabelle Préfontaine declares that they have no conflict of interest. Marc J. Lanovaz declares that he owns the intellectual property rights for the iSTIM.

Correspondence concerning this article should be addressed to Marc J. Lanovaz, École de Psychoéducation, Université de Montréal, C.P. 6128, succursale Centre-Ville, Montreal, QC, Canada, H3C 3J7.

Email: marc.lanovaz@umontreal.ca

Phone: 1 514-343-6111 \#81774 


\begin{abstract}
Although behavioral interventions have been known to effectively reduce stereotypy in children with ASD, these types of interventions are not accessible to all families. In response to this issue, we evaluated the effects of the iSTIM, an iOS application designed to support parents in the reduction of stereotypy in their child with $\mathrm{ASD}$. We used a series of $\mathrm{AB}$ designs to determine the effectiveness of the iSTIM on stereotypy using parents as behavior change agents. The use of iSTIM by the parents led to a reduction in stereotypy for six of seven participants. Our results suggest that the use of technology may be a cost effective and easily accessible method for parents to reduce stereotypy in their child with ASD.
\end{abstract}

Keywords: autism, behavioral interventions, parent training, stereotypy, technology. 


\section{Brief Report: Mobile Technology to Support Parents in Reducing Stereotypy}

Stereotypy is typically defined as the occurrence of repetitive and invariant behaviors that do not serve any apparent social function (Rapp \& Vollmer, 2005). As it represents one of the diagnostic subcriteria for the disorder, at least $50 \%$ of children with autism spectrum disorder (ASD) engage in some form of stereotypy (Melo et al., 2019). Stereotypy may directly interfere with learning, socialization as well as adaptive functioning, and lead to social stigmatisation (Coon \& Rapp, 2016; Lampi et al., 2018; Lanovaz et al., 2013). Although stereotypy may be a problem in some contexts, practitioners should not target stereotypy for reduction at all times with all children. Stereotypy should only be targeted for reduction in contexts in which its frequency is so high that it interferes with learning, social inclusion or daily functioning. For example, a child may engage in levels of vocal stereotypy so high (e.g., $80 \%$ of the time) that it prevents their participation in learning activities and significantly disrupts other students in the classroom. In this case, reducing vocal stereotypy may increase their level of functioning in the classroom while facilitating their social inclusion. On the other hand, a child who engages in stereotypy before bed or only briefly in class when excited should not be targeted for an intervention as it does not interfere with functioning.

Several behavioral interventions have strong empirical support for the reduction of stereotypy in children (Cook et al., 2018). Differential reinforcement (DR) and noncontingent reinforcement (NCR) are two effective interventions to reduce stereotypy in children with ASD (Rapp \& Lanovaz, 2016). The first intervention, DR, involves delivering a reinforcer (a) contingent on the occurrence of an alternative behavior (e.g., play) or (b) for periods of time wherein stereotypy is absent (Cividini-Mota et al., 2019; Weston et al., 2017). The second intervention, NCR, consists of providing a stimulus (music, toy or preferred food) that will 
provide the same stimulation as the stereotypy. However, behavioral interventions are not accessible to all families who need these services. Families may wait years to get access to effective publicly funded services in Canada (Ombudsman Ontario, 2016). In countries with private insurance schemes, these services are costly for those without insurance as they require the involvement of a professional for each child over several weeks (Wang \& Leslie, 2010). Accessibility to these services may also be a challenge for families who live in rural areas. A potential solution to the limitations of the current interventions offered to families would be to use technology to support parents in reducing stereotypy.

A notable example is I-Connect, developed by Wills and Mason (2014). This mobile application is conceived to support adolescents in self-regulating challenging behaviors in school. Crutchfield et al. (2015) evaluated the effects of I-Connect on stereotypy in two adolescents with ASD in their school environment. The authors reported that stereotypy had decreased for both participants after the introduction to the intervention offered by I-Connect. Though the app may be effective with adolescents with ASD who have adequate cognitive skills, many children with ASD do not have the ability to use a mobile application such as I-Connect independently to self-regulate and to self-evaluate their behaviors.

An alternative is the iSTIM (Préfontaine et al., 2019), which was designed to support parents in the reduction of stereotypy in their child with ASD. This application offers parents a solution and a method to reduce stereotypy in children who do not have the ability to use a selfmonitoring method. In their initial validation study, Préfontaine et al. (2019) had research assistants use the iSTIM with 11 children aged from 3 to 10 years old. The use of the iSTIM by the research assistants led to reductions in stereotypy in eight participants as well as an increase in functional engagement in four of those participants. The main limitation of the study was that 
the app was used by trained research assistants rather than parents. Parents have skill sets that differ from research assistants, which underlines the importance of replicating this study with parents. Consequently, the purpose of our study was to replicate and extend the study by Préfontaine et al. (2019) using parents as behavior change agents. Specifically, this research project aimed to determine whether parents were able to produce reductions in stereotypy by using the iSTIM.

\section{Method}

\section{Participants}

To recruit participants, we posted ads about our research project on Facebook and Instagram. We also recruited participants through centers providing services to children with developmental disabilities in [removed for blind review]. To take part in the study, children had to have received a diagnosis of ASD and engage in a high level of stereotypy. More specifically, the diagnosis of ASD had to be provided by an independent multidisciplinary team prior to their inclusion the study. Moreover, the children had to engage in one form of stereotypy at least $20 \%$ of the time in the context in which it occurred most often.

Twelve children with a prior diagnosis of ASD initially participated in the study with one of their parents. All parents provided informed consent and the research project was approved by our university research ethics board. Five participants withdrew from the study because either the parents did not have the time to continue the research project ( 2 participants) or stereotypy was occurring less than $20 \%$ of the time during baseline ( 3 participants). Thus, our sample was composed of seven children aged between two and nine years old. Each parent selected the context in which they wanted to reduce stereotypy as well as the form of stereotypy to target. Most parents selected to reduce stereotypy during free play and television watching as it 
hindered their child's participation in common social activities outside their home (e.g., participating in play dates, going to the movies). None of our participant were receiving other behavioral intervention services to reduce stereotypy during their participation in the study. Table 1 presents the characteristics of these participants, the context of intervention and the target behaviors. We used pseudonyms to identify each participant in order to preserve anonymity.

\section{Measures}

Severity of autistic symptoms. To characterize the severity of autistic symptoms in our participants, research assistants administered the Childhood Autism Rating Scale - Second Edition (CARS-2 ; Schopler, Van Bourgondien, Wellman, \& Love, 2010). This 15-item questionnaire uses a 4-point Likert scale to rate the severity of the participant's autistic symptoms ( 1 = no evidence of difficulty or abnormality to $3=$ severely abnormal $)$ based on direct observation and parental interviews. A high score represents a child with severe symptoms. The scores for CARS-2 allow to classify the children into 1 to 3 categories : minimum-to-no symptom of ASD (score between 15 and 29), mild-to-moderate symptoms of ASD (score between 30 and 36) and severe symptoms of ASD (score of 37 and over). This questionnaire has a good internal consistency with a Cronbach alpha of 0.94 .

Stereotypy and functional engagement. Research assistants directly measured the duration of stereotypy and functional engagement using the video recordings of each session. The definitions of the target behaviors for each participant are available in the Supplementary Materials (see Table A). Subsequently, a second observer measured at least $25 \%$ of the recordings for each participant to determine the interobserver agreement (IOA). To measure IOA, we used the block-by-block method (Mudford et al., 2009). This method involves dividing 
the observational period in 10-s intervals. The number of seconds in which the targeted behavior occurred is calculated for each interval. The smallest total of the two observers in a $10 \mathrm{~s}$ interval is then divided by the largest. The IOA is determined by calculating the mean of all intervals in each session. Table 2 (see Results) presents the mean IOAs for each participant.

Social validity. The social acceptability, ease of use and side effects of the intervention were measured using an adapted version of the Treatment Acceptability Rating Form-Revised (TARF-R; Reimers et al., 1991;). This 20-item questionnaire uses a five-point Likert scale $(1=$ lower social validity and $5=$ higher social validity). A score of 5 represents the most desirable outcome. For the following item: "How disruptive will it be to the family (in general) to carry out this treatment?", a score of 5 indicates that it would not be disruptive at all to carry out this treatment. This questionnaire has a good internal consistency with a Cronbach alpha of 0.92 and is one of the most used to assess social validity in behavioral research (Carter, 2007).

\section{iSTIM}

The iSTIM is an iOS application with four parent training and support modules, which is currently only available for research purposes. Each module begins with a training video teaching the parents how to implement the procedures which is then followed by a quiz to test their comprehension (except for module 1 which only involved responding to questions). Préfontaine et al. (2019) first described and designed the application to guide parents of children with ASD in the implementation of a personalized behavioral intervention to reduce stereotypy in their child. Figure 1 presents examples of screenshots for each module.

Module 1 - Preliminary Assessment. In module 1, the parents responded to eight questions regarding their child's stereotypy and the context in which it occurred. Thereupon, 
iSTIM recommended the data collection method, the preference assessment and the intervention that would be used by the parents.

Module 2 - Baseline measure. The second module involved measuring the baseline level of the child's targeted stereotypy. The application asked the parent to intervene as usual. Each baseline session lasted $10 \mathrm{~min}$. Depending on the responses to the questions in module 1, iSTIM recommended one of two data collection procedures: momentary time sampling or partial interval recording. For momentary time sampling, a visual timer appeared on the screen indicating the countdown for each 30-s interval. At the end of each interval, iSTIM asked the parent whether their child was engaging in stereotypy at that precise moment. For partial interval recording, iSTIM asked the parent whether their child engaged in stereotypy at least once during the previous interval. The application ended the module when the mobile application detected three consecutive sessions in which stereotypy was (a) stable, (b) not decreasing, and (c) between 20 and $80 \%$ (or after a maximum of 10 sessions).

Module 3 - Preference assessment. The third module guided parents to conduct a preference assessment to identify their child's preferred stimulus that was then used during the intervention. For this module, the application suggested one of two types of preference assessment: free-choice or paired-choice. The application first instructed the parents to preselect six stimuli according to their child's behavior, preference and age. For the free-choice assessment, the parents presented all six preselected stimuli to their child and let them freely engage with all of the stimuli. Every $30 \mathrm{~s}$, iSTIM asked the parents to identify the item with which their child was engaging with. In the paired-choice assessment, the application prompted the parents to present each possible pair of all six pre-selected stimuli and ask their child to choose only one. The parents indicated the selected item for each pair. The parents conducted 
two preference assessments on two different days. The iSTIM used the stimulus that was selected most frequently for the implementation of the intervention in the next module.

Module 4 - Implementation of the intervention. Based on the parental responses in the first module, the application recommended one of two interventions: DR and NCR. For DR, iSTIM prompted the parents every $30 \mathrm{~s}$ to provide the preferred stimulus (identified in Module 2) to their child if they were (a) not engaging in stereotypy and (b) engaging in an alternative appropriate behavior. In NCR, the application instructed the parents to provide unrestricted access to the preferred stimulus for the duration of each intervention session. Each session lasted 10 min. The iSTIM continued to prompt parents to collect data on stereotypy as they did during baseline. Once the application detected four consecutive sessions in which stereotypy had decreased under the mean of the baseline level, participation ended.

\section{Design and Procedures}

To assess the effects of the app, we conducted a series of $A B$ quasi-experiments wherein each participant served as their own control. That is, a research assistant measured stereotypy before and during the implementation of the intervention while the parent was using the iSTIM. We employed this research design because (a) its implementation did not require the withdrawal of the intervention once implemented and (b) we did not have enough devices to implement the intervention with all participants simultaneously preventing the use of a multiple-baseline design. Our prior experience with single-case designs raised concerns that parents would be more likely to withdraw from the study if they were asked to stop an intervention that was effective or to wait several months before starting the intervention. Families participated in sessions once or twice per week over a period of 8 to 16 weeks. During each session, a research assistant provided an iPod to the parent on which the iSTIM was installed and instructed the parents to use the app. 
The app was only available for iOS devices (e.g., iPods, iPhones), which prevented the use of Android phones. Furthermore, the research team used iPods for the research project because they were less expensive than iPhones and iPads. The parents used the iSTIM with their child independently while the research assistants recorded each session on video. To isolate the effects of the iSTIM, the research assistants never responded to the parent questions regarding the use of the application. If a parent asked a question regarding the app, the research assistant told them to do the best that they could with information provided by the iSTIM.

\section{Analysis}

To examine the effects of the intervention, we used the dual-criteria method developed by Fisher et al. (2003). This method consists in generating a regression slope as well as a constant from the mean of the baseline data and to overlap the two lines over the intervention phase. Then, the binomial test allows to determine the number of points that should be bellow the two lines to rule out the possibility that the observed effect is the result of chance. The method has been shown to provide adequate control over Type I errors produced during the analysis of single-case graphs (Falligant et al., 2019; Lanovaz et al., 2017), which is why we selected it to analyze the results of the current study.

\section{Results}

Table 2 presents the mean levels of stereotypy and functional engagement during baseline as well as during the implementation of the intervention for each participant. Figures 2 and 3 depict the percentage of stereotypy (left panels) and functional engagement (right panels) during baseline and during intervention. When compared to baseline using the dual-criteria analysis, the parents using the iSTIM produced reductions in stereotypy for all but one participant (i.e., Peter). A decreasing pattern during baseline prevents us from drawing clear conclusions for Peter (see 
third panel of Figure 3). The dual-criteria analysis suggests that the app had mixed effects on functional engagement: Functional engagement increased for Alex and Kyle, remained stable for Kim, Matt and Peter, and decreased for Brian. The graph shows an increasing trend for Carl (see second panel of Figure 3), but the initial points of the intervention phase are too low to produce significant results. The average score on the social validity measure, the TARF-R, was 4.3 on a 5-point scale. Nearly all individual items had mean scores above 4, indicating high social validity. The Supplementary Materials (see Table B) include a table for average scores on each individual item of the TARF-R. That said, the scores may have been lower if we had not provided the devices to the families. Otherwise, some families may have indicated that the treatment was not affordable and not accessible because iOS devices can be expensive.

\section{Discussion}

The use of the iSTIM by the parents led to a reduction in stereotypy in six of seven participants in various contexts. This study replicates and extends Préfontaine et al. (2019) by showing that parents could effectively use the iSTIM to decrease stereotypy in children with ASD. Furthermore, our results are also consistent with previous research regarding the effectiveness of the use of technology (Wills \& Mason, 2014; Crutchfield et al., 2015) and the involvement of parents as behavior change agents (e.g., Gerow et al., 2019) to reduce stereotypy in individuals with ASD. However, parents using the iSTIM only increased functional engagement in two participants. Two hypotheses may explain these results. First, some children already had very high levels of functional engagement at baseline (i.e., Brian and Peter), making increases in functional engagement nearly impossible. Second, interventions to teach appropriate behavior such as play typically take more than four to six intervention sessions to produce 
increases in behavior (Quigley et al., 2018). Therefore, the parents may need to use the iSTIM for longer periods of time in order to produce meaningful changes in functional engagement.

To our knowledge, iSTIM is the only iOS application designed to support parents in the reduction of stereotypy. Our results indicate that a mobile application used by parents can teach them how to implement behavioral interventions to effectively reduce stereotypy. From a practical standpoint, our results indicate that more families could have access to effective services to reduce stereotypy in their child with ASD. The use of technology and parents as behavioral change agents eliminates issues regarding geographical isolation and waiting lists by increasing accessibility to all families in need of services. This method is also cost-effective since it does not require a professional being present during multiple sessions.

Our study has some limitations that should be discussed. First, we used a quasiexperimental $\mathrm{AB}$ design. This design does not rule out the effects of historical and maturational variables. Despite this limitation, the replication of the intervention effects across six participants with variable baseline lengths suggests that the intervention, and not some confounding variable, was responsible for the observed changes. Second, the children did not participate in a functional analysis to identify the function of their stereotypy. Therefore, we cannot be certain that the behaviors did not serve any apparent social function as defined by Rapp and Vollmer (2005). To counter this limitation, the iSTIM asked the parent if the behavior persisted when the child was alone in the first module, a common indicator of a non-social function. The parents of all participants reported that stereotypy persisted when their child was alone. The presence of an observer may also have caused some bias in our results. To minimize this concern, research assistants conducted three observation sessions before introducing iSTIM. Participants and their 
parents could thus get accustomed to the presence of the research assistant prior to formal data collection.

Moreover, two participants withdrew from the study due to a lack of time to implement the procedures. These withdrawals may have biased our results as some parents may stop using the app when they feel that the intervention does not meet their needs. Researchers should investigate this issue in the future to increase the number of users who may benefit from iSTIM. Additionally, the study did not compare the effects of iSTIM with other types of common behavioral interventions and did not include a placebo condition, which indicates that our data needs to be interpreted with caution. Finally, we did not measure generalization and maintenance of the behavior changes over time, which would provide important information on the effects and side-effects of the iSTIM. Future research should address the aforementioned limitations by replicating our study within a large randomized control trial that would measure stereotypy in multiple contexts and implement more rigorous pre-experimental assessments. Such replication would be essential to support the potential utility of the iSTIM in practical settings.

\section{Compliance with Ethical Standards}

Funding: This study was funded by [removed for blind review]

Ethical Approval: All procedures performed in this study were in accordance with the ethical standards of [removed for blind review] and with the 1964 Helsinki declaration and its later amendments.

Informed Consent: Parents provided informed consent for them and their child.

Conflict of Interest: [removed for blind review] 


\section{References}

American Psychiatric Association (2013). Diagnostic and statistical manual of mental disorders: DSM-5 (5th ed.). Washington, DC: American Psychiatric Association.

Carter, S. L. (2007). Review of recent treatment acceptability research. Education and Training in Developmental Disabilities, 42(3), 301-316.

Cividini-Mota, C., Garcia, A. R., Livingston, C., \& MacNaul, H. L. (2019). The effect of response interruption and redirection with and without a differential reinforcement of alternative behavior component on stereotypy and appropriate responses. Behavioral Interventions, 34(1), 3-18. https://doi.org/10.1002/bin.1654

Cook, J. L., Rapp, J. T., \& Brogan, K. M. (2018). Assessment and treatment of stereotypical behavior displayed by children with autism spectrum disorders. In C. Bodiford McNeil, L. Borduin Quetsch, \& C. M. Anderson (Eds.), Handbook of parent-child interaction therapy for children on the autism spectrum (pp. 147-168). Springer.

Coon, J. C., \& Rapp, J. T. (2019). Brief report: Evaluating college students' perceptions of a child displaying stereotypic behaviors: Do changes in stereotypy levels affect ratings? Journal of Autism and Developmental Disorders. Advance online publication. https://doi.org/10.1007/s10803-019-03916-2

Crutchfield, S.A., Mason, R.A., Chambers, A., Wills, H. P., \& Mason, B. A. (2015). Use of a selfmonitoring application to reduce stereotypic behavior in adolescents with autism: A preliminary investigation of I-Connect. Journal of Autism and Developmental Disorders, 45, 1146-1155. https://doi.org/10.1007/s10803-014-2272-x 
Falligant, J. M., McNulty, M. K., Hausman, N. L., \& Rooker, G. W. (2019). Using dual-criteria methods to supplement visual inspection: Replication and extension. Journal of Applied Behavior Analysis. Advance online publication. https://doi.org/10.1002/jaba.665

Fisher, W. W., Kelley, M. E., \& Lomas, J. E. (2003). Visual aids and structured criteria for improving visual inspection and interpretation of single-case designs. Journal of Applied Behavior Analysis, 36(3), 387-406. https://doi.org/10.1901/jaba.2003.36-387

Gerow, S., Rivera, G., Akers, J. S., Kirkpatrick, M., \& Radhakrishnan, S. (2019). Parentimplemented treatment for automatically maintained stereotypy. Behavioral Interventions, 34(4), 466-474. https://doi.org/10.1002/bin.1689

Lampi, A., Fitzpatrick, P., Romero, V., Amaral, J., \& Schmidt, R. C. (2018). Understanding the influence of social and motor context on the co-occurring frequency of restricted and repetitive behaviors in autism. Journal of Autism and Developmental Disorders. Advance online publication. https://doi.org/10.1007/s10803-018-3698-3.

Lanovaz, M. J., Huxley, S. C., \& Dufour, M. M. (2017). Using the dual-criteria methods to supplement visual inspection: An analysis of nonsimulated data. Journal of Applied Behavior Analysis, 50(3), 662-667. https://doi.org/10.1002/jaba.394

Lanovaz, M. J., Robertson, K. M., Soerono, K., \& Watkins, N. (2013). Effects of reducing stereotypy on other behaviors: A systematic review. Research in Autism Spectrum Disorders, 7(10), 1234-1243. https://doi.org/10.1016/j.rasd.2013.07.009

Melo, C., Ruano, L., Jorge, J., Ribeiro, T. P., Oliveira, G., Azevedo, L., \& Temudo, T. (2019). Prevalence and determinants of motor stereotypies in autism spectrum disorder: A systematic review and meta-analysis. Autism. Advance online publication. https://doi.org/10.1177/1362361319869118 
Mudford, O. C., Taylor, S. A., \& Martin, N. T. (2009). Continuous recording and interobserver agreement alogorithms reported in the Journal of Applied Behavior Analysis (19952005). Journal of Applied Behavior Analysis, 42(1), 165-169. https://doi.org/10.1901/ jaba $.2009 .42-165$

Ombudsman Ontatrio. (2016). Nowhere to turn: Investigation into the Ministry of Community and Social Services' response to situations of crisis involving adults with developmental disabilities Retrieved from https://www.ombudsman.on.ca/Files/sitemedia/Documents/ NTT-Final-EN-w-cover.pdf

Préfontaine, I., Lanovaz, M. J., McDuff, E., McHugh, C., \& Cook, J. (2019). Using mobile technology to reduce engagement in stereotypy: A validation of decision-making algorithms. Behavior Modification, 43(2), 222-245. https://doi.org/10.1177/0145445517748560

Quigley, J., Griffith, A. K., \& Kates-McElrath, K. (2018). A comparison of modeling, prompting, and a multi-component intervention for teaching play skills to children with developmental disabilities. Behavior Analysis in Practice, 11(4), 315-326. https://doi.org/10.1007/s40617-018-0225-0

Rapp, J. T., \& Lanovaz, M. J. (2016). Stereotypy. In Singh, N. N. (Ed.), Clinical handbook of evidence-based practices for individuals with intellectual and developmental disabilities. New York, NY: Springer. https://doi.org/10.1007/978-3-319-26583-4_28

Rapp, J. T., \& Vollmer, T. R. (2005). Stereotypy I: A review of behavioral assessment and treatment. Research in Developmental Disabilities, 26(6), 527-547. https://doi.org/10.1016/j.ridd.2004.11.005 
Reimers, T. M., Wacker, D. O., \& Cooper, L. J. (1991). Evaluation of the acceptability of treatments for children's behavioral difficulties: Ratings by parents receiving services in an outpatient clinic. Child \& Family Behavior Therapy, 13(2), 53-71. https://doi.org/10.1300/J019v13n02_04

Schopler, E., Van Bourgondien, M., Wellman, J., \& Love, S. (2010). Childhood autism rating scale—Second edition (CARS2): Manual. Los Angeles: Western Psychological Services.

Wang, L., \& Leslie, D. L. (2010). Health care expenditures for children with autism spectrum disorders in Medicaid. Journal of the American Academy of Child and Adolescent Psychiatry, 49(11), 1165-1171. https://doi.org/10.1016/j.jaac.2010.08.003

Weston, R., Hodges, A., \& Davis, T. N. (2017). Differential reinforcement to treat challenging behavior among children with autism: A systematic and quality review. Behavior Modification, 42(4), 584-609. https://doi.org/10.1177/0145445517743487

Wills, H. P., \& Mason, B. A. (2014). Implementation of a self-monitoring application to improve on-task behavior: A high school pilot study. Journal of Behavioral Education, 23, 421434. https://10.1007/s10864-014-9204-X 


\section{Table 1}

Participant Characteristics

\begin{tabular}{|c|c|c|c|c|c|}
\hline Names & Age & $\begin{array}{l}\text { CARS-2 } \\
\text { T-score }\end{array}$ & Context & Stereotypy & $\begin{array}{c}\text { Functional } \\
\text { engagement }\end{array}$ \\
\hline Alex & 5 & 60 & $\begin{array}{l}\text { Television } \\
\text { watching }\end{array}$ & $\begin{array}{l}\text { Pacing and } \\
\text { jumping }\end{array}$ & $\begin{array}{l}\text { Appropriate play and } \\
\text { television watching }\end{array}$ \\
\hline Kim & 2 & 61 & Free play & Mouthing & $\begin{array}{l}\text { Appropriate use of the } \\
\text { oral stimulator }\end{array}$ \\
\hline Matt & 4 & 54 & Free play & Vocal stereotypy & Appropriate play \\
\hline Carl & 9 & 34 & Morning routine & Body rocking & $\begin{array}{l}\text { Engagement in the } \\
\text { routine }\end{array}$ \\
\hline Brian & 5 & 34 & Free play & Mouthing & Appropriate play \\
\hline Kyle & 4 & 49 & $\begin{array}{l}\text { Television } \\
\text { watching }\end{array}$ & $\begin{array}{l}\text { Pacing and } \\
\text { jumping }\end{array}$ & Sitting appropriately \\
\hline Peter & 8 & 61 & Free play & Vocal stereotypy & Appropriate play \\
\hline
\end{tabular}

Note. CARS-2 = Childhood Autism Rating Scale-Second Edition (Schopler et al., 2010). 


\section{Table 2}

Means for Stereotypy, Functional Engagement, and Interobserver Agreement

\begin{tabular}{lccccccc}
\hline & \multicolumn{3}{c}{ Stereotypy $(\%)$} & & \multicolumn{3}{c}{ Functional engagement $(\%)$} \\
\cline { 2 - 3 } \cline { 7 - 8 } Name & Baseline & Intervention & IOA & & Baseline & Intervention & IOA \\
\hline Kim & 51.8 & 10.8 & 88.1 & & 8.1 & 61.5 & 86.3 \\
Matt & 41.6 & 2.5 & 91.4 & & 0.0 & 12.0 & 100.0 \\
Carl & 79.7 & 3.5 & 90.3 & & 36.3 & 49.3 & 88.3 \\
Brian & 29.0 & 0.0 & 94.9 & & 83.9 & 79.0 & 88.1 \\
Kyle & 19.0 & 0.8 & 88.0 & & 13.6 & 78.2 & 84.3 \\
Peter & 19.8 & 1.3 & 91.0 & & 72.8 & 80.0 & 95.1 \\
\hline
\end{tabular}

Note. IOA = interobserver agreement. 


\section{Figure 1}

Screenshot Examples for Each Module of the iSTIM

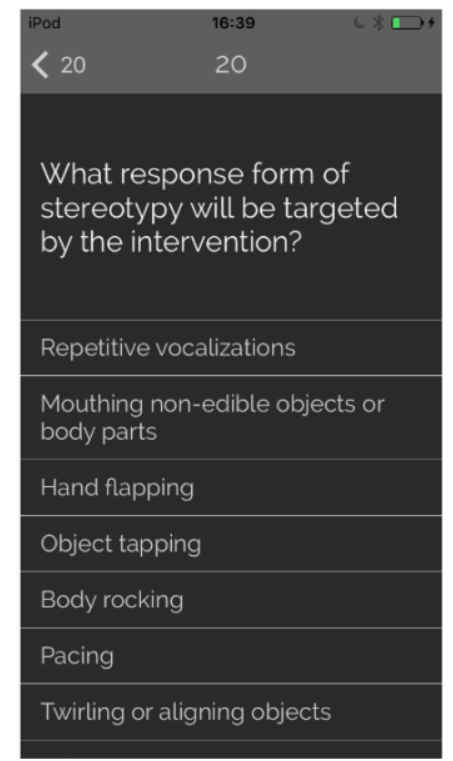

Module 1

(Question 2)

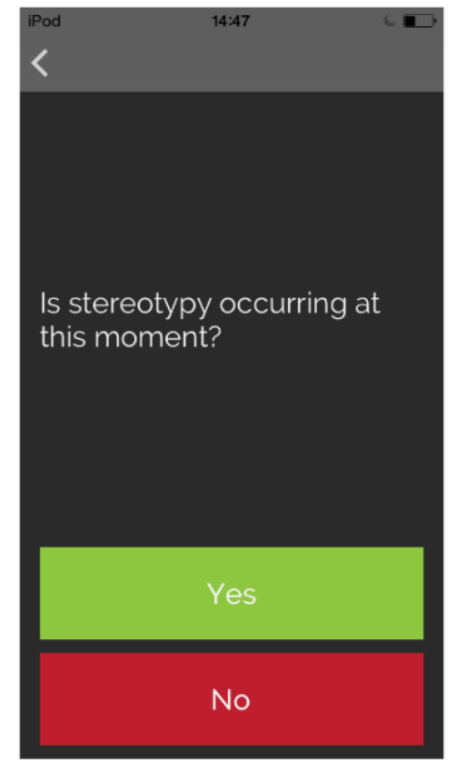

Module 2 (momentary time sampling)

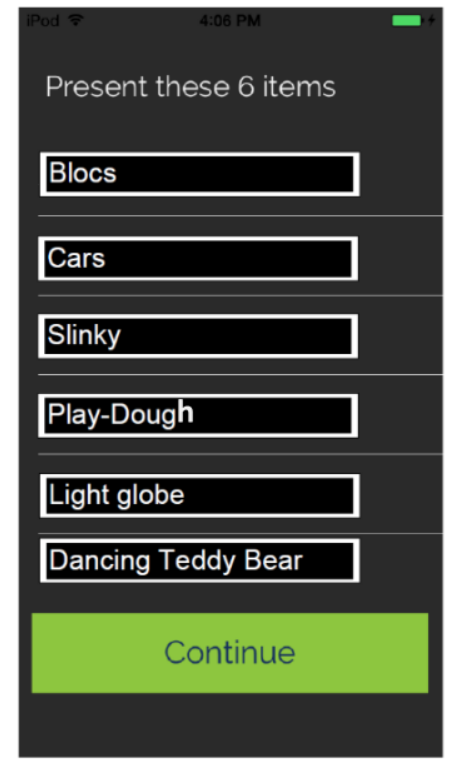

Module 3

(free-choice assessment)

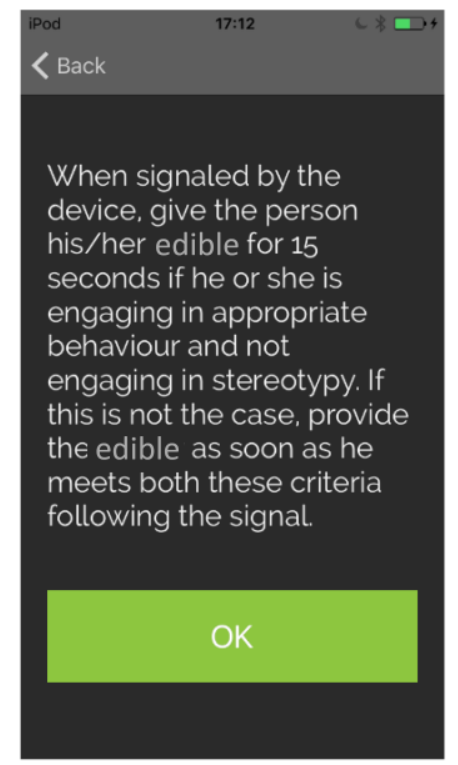

Module 4 (differential reinforcement)
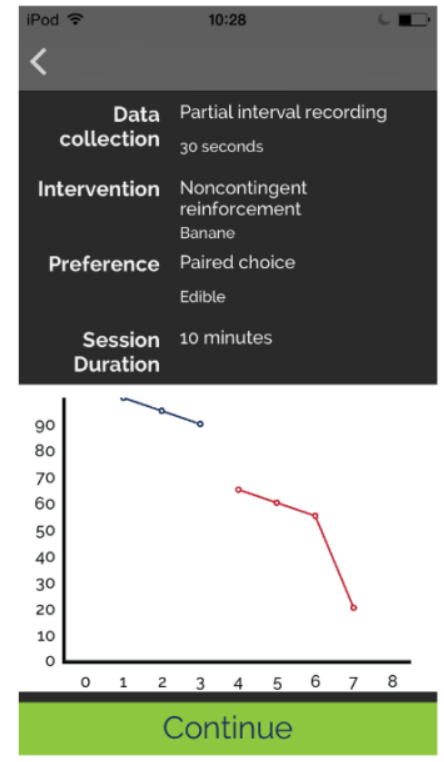

Summary screen

Reprinted and adapted with permission from "Using mobile technology to reduce engagement in stereotypy: A validation of decision-making algorithms" by I. Préfontaine, M. J. Lanovaz, E. McDuff, C. McHugh, and J. L. Cook, 2019, Behavior Modification, 43, p. 228.

(https://doi.org/10.1177/0145445517748560) (C) The Authors. 


\section{Figure 2}

Percentage of Time Engaged in Stereotypy and Functional Engagement During Baseline and iSTIM Sessions

Stereotypy
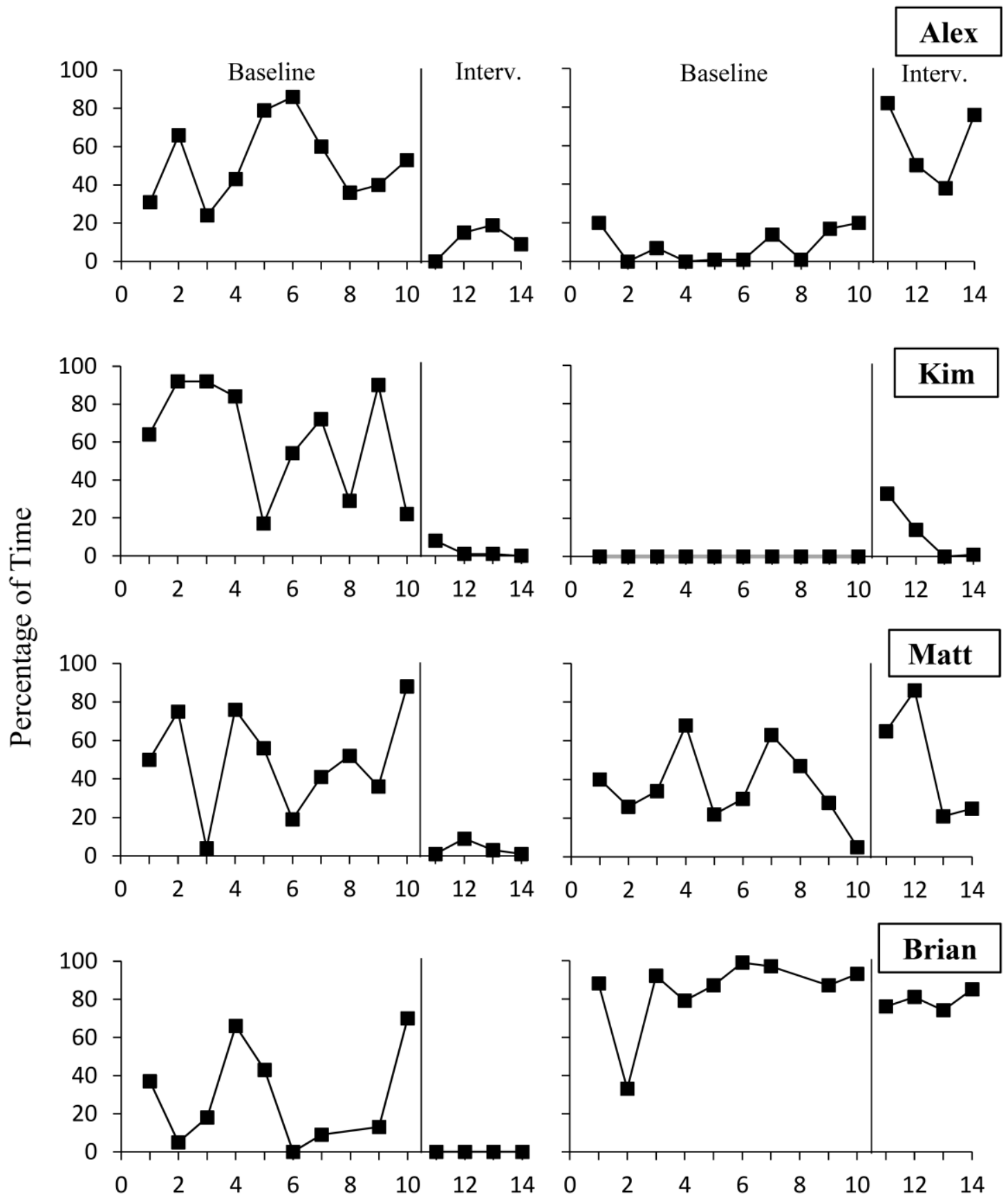

Functional Engagement
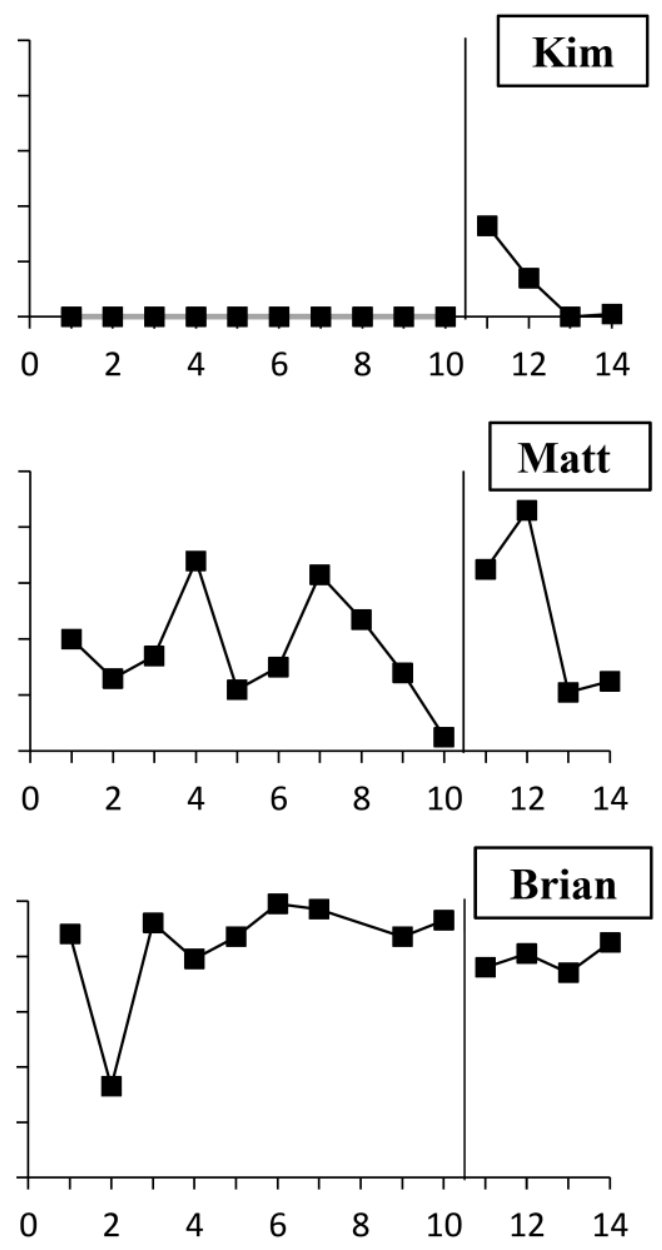

Sessions 


\section{Figure 3}

Percentage of Time Engaged in Stereotypy and Functional Engagement During Baseline and iSTIM Sessions

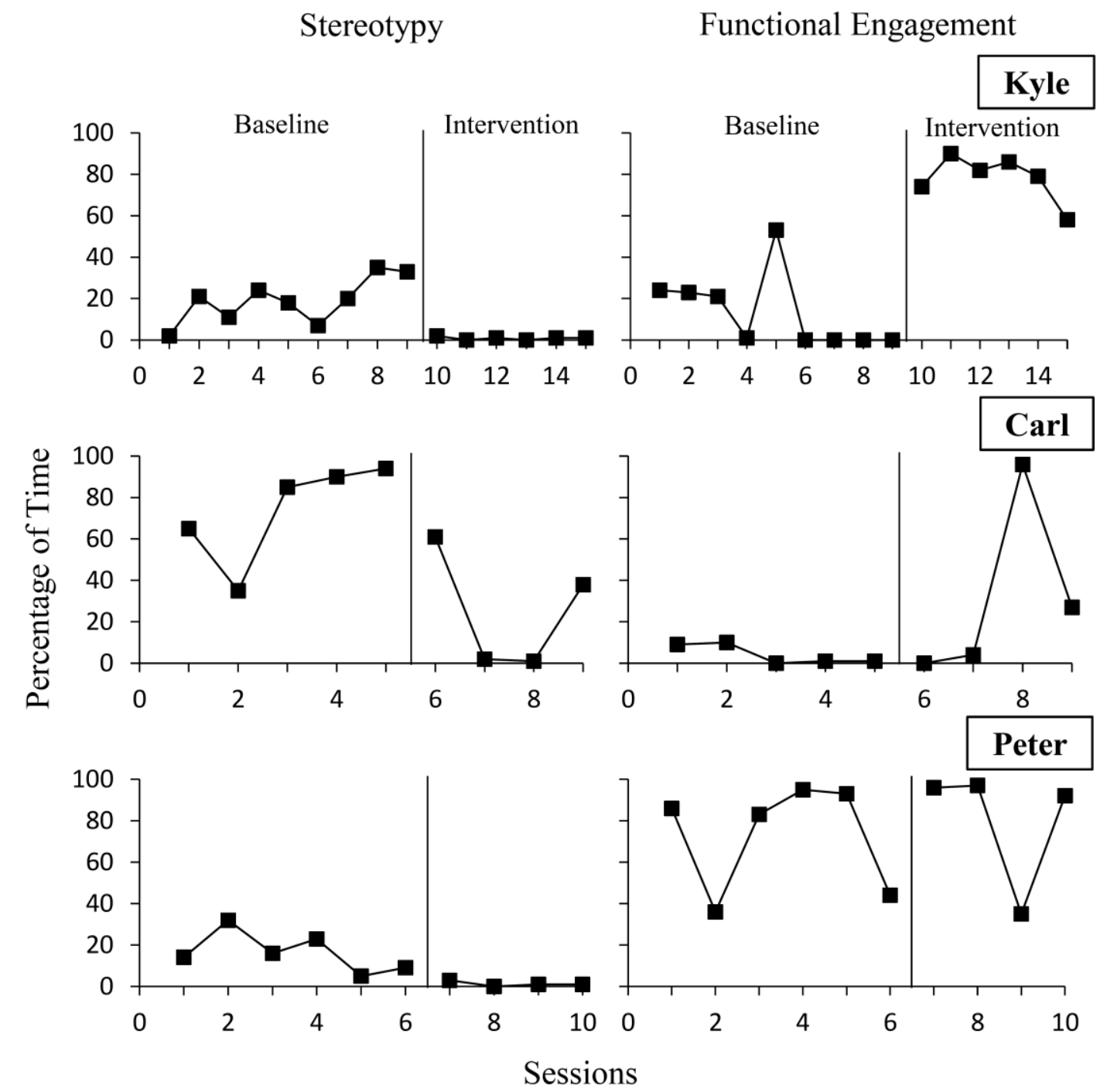

